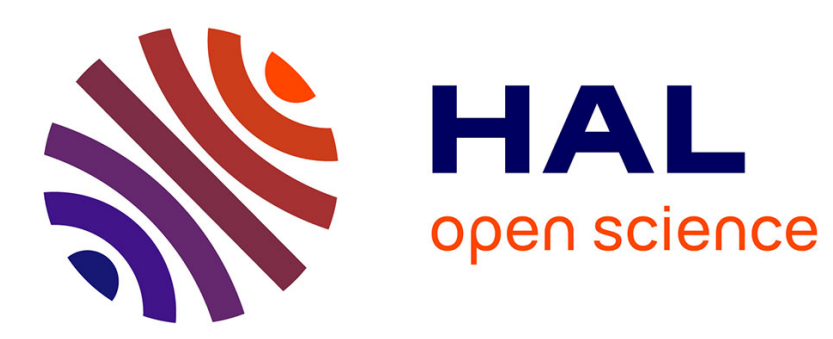

\title{
An acoustoelectric-induced tailorable coupled resonator surface acoustic waveguide
}

\author{
Farshid Taleb, Sara Darbari, Abdelkrim Khelif, H Taleb
}

\section{To cite this version:}

Farshid Taleb, Sara Darbari, Abdelkrim Khelif, H Taleb. An acoustoelectric-induced tailorable coupled resonator surface acoustic waveguide. Journal of Physics D: Applied Physics, 2021, 54 (22), pp.225301 (12). hal-03549531

\author{
HAL Id: hal-03549531 \\ https://hal.science/hal-03549531
}

Submitted on 31 Jan 2022

HAL is a multi-disciplinary open access archive for the deposit and dissemination of scientific research documents, whether they are published or not. The documents may come from teaching and research institutions in France or abroad, or from public or private research centers.
L'archive ouverte pluridisciplinaire HAL, est destinée au dépôt et à la diffusion de documents scientifiques de niveau recherche, publiés ou non, émanant des établissements d'enseignement et de recherche français ou étrangers, des laboratoires publics ou privés. 


\title{
An acoustoelectric-induced tailorable coupled resonator surface acoustic Waveguide
}

\author{
F. Taleb ${ }^{1}$ and S. Darbari ${ }^{1}$, A. Khelif ${ }^{2}$, and H. Taleb ${ }^{3}$ \\ ${ }^{1}$ Nano Sensors and Detectors Lab., Nano Plasmophotonic Research Group, Faculty of Electrical and Computer Engineering, Tarbiat \\ Modares University, Tehran 1411713116, Iran \\ ${ }^{2}$ FEMTO-ST Institute, UBFC, CNRS, ENSMM, UTBM, 15B Avenue des Montboucons, 25030 Besançon, France \\ ${ }^{3}$ Advanced Devices Simulation Lab., Nano Plasmophotonic Research Group, Faculty of Electrical and Computer Engineering, \\ Tarbiat Modares University, Tehran 1411713116, Iran
}

E-mail: s.darbari@modares.ac.ir

\begin{abstract}
Here, we introduce a Tailorable coupled resonator surface acoustic waveguide (CRSAW), based on a line defect of elliptical cylinders inside a phononic crystal $(\mathrm{PnC})$ of $\mathrm{ZnO}$ pillars over a Si substrate. The designed elliptical resonators allow emerging of a lowly dispersive, single mode shear guiding band inside the local resonance bandgap, owing to their partially broken structural symmetry in comparison with their previously reported counterparts such as pillars and hollow cylinders. Moreover, to introduce reconfigurable waveguiding behavior, we benefit from the acoustoelectric-induced elasticity modulation of $\mathrm{ZnO}$, as a semiconducting piezoelectric. Switching the conductivity of $\mathrm{ZnO}$ structures between two limiting low and high values $(0.01 \mathrm{~S} / \mathrm{m}$ to $100 \mathrm{~S} / \mathrm{m})$, a considerable waveguiding modulation in FWHM $(\Delta \mathrm{FWHM}=-21 \%)$ and guiding frequency $\left(\Delta f_{d}=-2.81 \%\right)$ are achieved for the designed optimized elliptical cylinder CRSAW, while maintaining acceptable loss value. Benefiting from the proposed ZnO-based elliptical CRSAW, we have achieved simultaneous single mode and low bandwidth surface acoustic waveguiding behavior, without involving significant fabrication complications. The designed structures open up new horizons towards realization of promising building blocks for designing reconfigurable, efficient, and miniature SAW RFfilters, demultiplexers, and Mach-Zehnder devices for wireless communication applications.
\end{abstract}

Keywords: Phononic Crystal, SAW, CRSAW, Acoustoelectric

\section{Introduction}

Implementation of surface acoustic wave (SAW) devices [1-9] into phononic crystals (PnC), which have found applications in areas such as acoustic waveguides, resonators, filters, vibration reduction, demultiplexers and sensors [10-26], can combine the advantages of both domains, thus providing new options to control the propagation of SAWs and create new fields of applications in SAW devices e.g. RF-filter and duplexers. For instance, applying a PnC as a basic platform for the RF-SAW filters can lead to better localization of the elastic wave to the waveguiding design and better filtering performance. Thus, SAW PnCs, as repetitive arrays of inclusions capable of opening bandgaps for incident SAWs, have recently attracted attention of researchers [27-32]. Moreover, the local resonance bandgaps, which occur at frequency ranges lower than those of Bragg bandgaps in PnCs of local resonators have been the topic of many recent theoretical and experimental studies for SAWs [33-39]. In this regard, a defected local resonance PnC can be used to realize miniature high performance SAW devices such as waveguides and resonators [40-43].

Propagation of an elastic wave inside a piezoelectric medium creates an extra elastic stiffness, due to the coupled piezoelectric effect induced electric field. This electric field can be screened by adding extra charge carriers to the media, thus changing the elastic constant effectively. In other words, whenever an elastic wave 
travels through a piezoelectric medium, an additional effective stiffness emerges, which can be relaxed by increasing the carrier concentration or conductivity (by UV illumination and etc.), and affects the elastic wave velocity and phase [44]. This phenomenon, known as acoustoelectric effect (AE), was firstly studied in 1953 [45], and is mostly applied to realize UV SAW-based detectors [46-48]. AE can be utilized to induce effective changes in the elasticity parameters of the piezoelectric materials, leading to achieve a tunable behavior in piezoelectric-based SAW devices, consequently. The proposed AE-assisted mechanism for designing reconfigurable SAW devices benefits from low cost, and technological compatible piezoelectric/semiconducting layers like $\mathrm{ZnO}$, as compared with the previously reported reconfigurable SAW devices [49,50]. Moreover, the proposed AE-assisted reconfigurable devices can be packed in small dimensions, using a simple, rapid, and contact-less tuning mechanism, such as UV-illumination. Regarding this, in our previous report, we have studied a SAW PnC, constituted of ZnO pillars in nano-dimensions, in which line defects of hollow cylinders were used to achieve SAW waveguiding behavior in GHz frequency range and AE was utilized to create a tunable waveguiding behavior [51]. Recently, we have also reported a SAW demultiplexing behavior based on local resonance waveguides, in which hollow cylinder waveguides with different outer and inner radii serve as the input and output channels of the multiplexer [52]. We have shown that hollow cylinder resonators allow good controllability over their guiding frequency and transmission bandwidth by changing their structural parameters, such as inner and outer radii. However, these resonators create multiple guiding modes inside the local resonance bandgap, which is not suitable for designing low loss and single mode waveguides and demultiplexing channels. Additionally, the fabrication of lowly dispersive hollow cylinders with very thin walls (nanometer dimensions) can be challenging, leading to difficulty in achieving the precise desired structural parameters and reliability.

To overcome these limitations, in this study, we have introduced a novel acoustic waveguide, consisting of acoustically coupled elliptical cylinders. By optimizing the structural parameters of this line defect into a $\mathrm{PnC}$ of $\mathrm{ZnO}$ pillars, we have observed emerging of a single guiding mode with low group velocity inside the local resonance bandgap. Also from the fabrication point of view, fabricating the elliptical cylinders in low dimensions is much easier in comparison with fabrication of thinned hollow cylinder resonators with comparable frequency dispersion. The guiding mode transmission created by the designed elliptical cylinder exhibits a value of full width at half maximum (FWHM), which is comparable with that of the designed hollow cylinders, studied in our previous report [51]. To further reduce the group velocity of the guiding mode and the FWHM of the elliptical cylinder waveguide, hereby we have utilized the coupled resonator acoustic waveguide (CRAW) structure for the SAWs. CRAWs have been recently investigated in elastic/acoustic bulk wave domains [53-57], after the introduction of their analogous in optical waves domain known as coupled resonator optical waveguides (CROW) [58-61]. In a CRAW setup, the spacing between the defect resonators can be changed to control the group velocity and pass-band of the CRAW through evanescent field coupling of defect resonators. From physical point of view, in a CRAW, as the spacing between resonators increases, the evanescent displacement field coupling between the nearest neighbor resonators decreases, thus the group velocity of the guiding mode reduces since it has a direct relationship with the resonators field coupling [54]. Same phenomenon can occur in a coupled resonator surface acoustic waveguide (CRSAW), in which separating of waveguide resonators gradually eliminates the displacement field coupling among the resonators, thus flattening the guiding band dispersion and decreasing the related transmission bandwidth. Studying the hollow cylinders and elliptical cylinders in the CRSAW form, we have shown a significant improvement in the waveguide bandwidth. The amount of spacing between the defect resonators can be changed to tune the SAW waveguide/filter bandwidth and increase the quality factor. The 
studied low bandwidth, high quality factor CRSAWs are proposed as promising CMOS-compatible platforms to realize high quality factor RF-filters, demultiplexers and also acoustic wave isolators.

\section{Physical principles and structure}

In a phononic crystal (PnC) platform, consisting of a pillar array on a solid substrate, each pillar can behave as a local resonator, when exposed to surface acoustic waves with specific frequencies lower than Brag frequencies. These local resonances can be coupled to the surface modes of the substrate, leading to emergence of surface coupled modes, resulting in emergence of local resonance guiding bands. The dispersion of these individual local resonance bands can be changed by the geometry and structural parameters of the pillars, so that forbidden gaps can open between them, the gaps which are called local resonant bandgaps in the literature [33-43].

Tailoring a line defect inside a PnC, consisting of resonators, we can create local resonance guiding modes inside the local resonance gap. Benefiting from low frequency local resonance gap is suitable for designing miniature SAW waveguides/filters with transmission characteristics correlated with the shape and the structural parameters of the defect resonators. To achieve a single mode shear waveguide, we break the structural symmetry of the defect resonators in the wave propagation plane by introducing elliptical cylinder line defect, as shown schematically in figure 1. This type of defect can create a single guiding mode inside the bandgap of the $\mathrm{PnC}$, but the created guiding band is more dispersive than those of the previously reported hollow cylinder line defect [49]. In order to reduce the group velocity of the elliptical defect guiding band, we have implemented the CRSAW structure in which the extra spacing between the defect resonators can determine the coupling strength between the resonators, the guiding bandwidth and the group velocity, consequently. To introduce tunable waveguiding behavior and further bandwidth control, AE is applied through changing the conductivity of the $\mathrm{ZnO}$ structures and changing their effective elasticity. This effect can be taken into action by utilizing UV illumination and increase the $\mathrm{ZnO}$ structures conductivity. The elliptical cylinder CRSAWs can be used as high quality factor SAW waveguides/filters with controllable transmission bandwidth and frequency that can find applications in field of RF communications.

The prepared scheme of the proposed high quality factor CRSAW, in addition to its top view, are shown in figure 1. As shown in this figure, SAW is fed into the $\mathrm{PnC}$, which is made of $\mathrm{ZnO}$ pillars in a square lattice form over a thick Si substrate. The CRSAW is consisted of defect resonators (in this case, elliptical cylinders) with extra spacing among them. The desired SAW is triggered through a source cutline in front of the device and the output SAW is detected over an output cutline. 


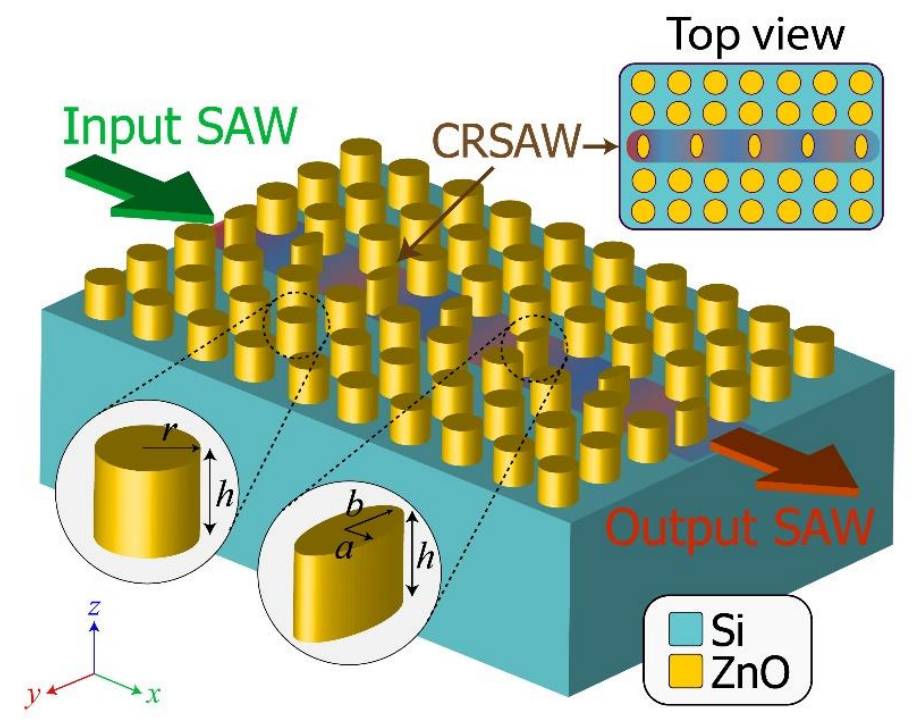

Figure 1. The prepared scheme of the proposed high quality factor CRSAW.

\section{Physical principles and equations}

Atomic scale displacement, stress and strain are created due to the propagation of elastic waves inside a solid material. Considered as a type of elastic waves, SAW propagation conforms to the elastic wave equations. So, to evaluate SAW propagation in a linear elastic media, as Si, the coupled equations (1), and (2) should be solved simultaneously [55]:

$$
\begin{array}{lr}
S_{i j}=\frac{1}{2}\left(\partial_{j} u_{i}+\partial_{i} u_{j}\right) & i, j=x, y, z \\
T_{i j}=c_{i j} S_{i j} & i, j=x, y, z
\end{array}
$$

In these equations $\mathbf{S}, \mathbf{u}, \mathbf{T}$, and $c$ are the strain, the atomic displacement, the stress, and the elastic matrix of the media.

In piezoelectric materials, an electric field is generated due to the piezoelectric effect and the elastic wave induces mechanical deformations. Thus, the stress, strain, electrical field and electrical displacement fields are coupled to each other through equations (3) and (4) [55]:

$$
\begin{aligned}
& {[T]_{6 \times 1}=[C]_{6 \times 6}[S]_{6 \times 1}-[e]_{6 \times 3}^{T}[E]_{3 \times 1}} \\
& {[D]_{3 \times 1}=[e]_{3 \times 6}[S]_{6 \times 1}-[\varepsilon]_{3 \times 3}[E]_{3 \times 1}}
\end{aligned}
$$

wherein $e, \varepsilon, \mathbf{D}$ and $\mathbf{E}$ are the piezoelectric coupling matrix, dielectric matrix, electric displacement and electric field, respectively.

Moreover, elastic wave equation in frequency domain follows equation (5):

$$
-\rho \omega^{2} \mathrm{u}=\nabla \cdot T
$$


wherein $\rho$, and $\omega$ are density, and frequency respectively. It is notable that the numerical solutions of the discussed equations are used to calculate transmission spectra (equations (1-4)), and elastic energy band structure curves (equation (5)). The given set of equations is solved numerically by a 3D finite element method.

Moreover, to investigate the effect of excess carriers on the elastic wave propagation in the piezoelectric materials, we should take in to account the acoustoelectric effect in the wave equation. Excess carriers in a semiconducting piezoelectric material can screen the effect of mechanically induced electrical field. This field is created due to the piezoelectric effect and can increase the elasticity of the material effectively, changing the velocity and amplitude of a travelling elastic wave. UV illumination can be utilized to generate excess carriers in such materials, thus controlling the elastic constant of the material. This interaction can be useful in inducing tenability in the frequency and mechanical characteristics of the devices made from semiconductor piezoelectric materials. Whenever the amount of excess carriers is compared to the intrinsic carrier concentration, the electrical field is related to the electrical displacement through the equation (6) [44]:

$$
[D]_{3 \times 1}=\frac{-j}{\omega} \sigma[E]_{3 \times 1}
$$

Adding the given equation to equation (4), a new set of equations for the propagation of elastic waves inside a semiconductor piezoelectric material in presence of acoustoelectric effect is driven. Stress matrix will be related to the strain matrix through an effective elastic constant matrix $c^{\prime}$ whose elements depend on the materials conductivity $(\sigma)$, dielectric constant $(\varepsilon)$, frequency of the traveling wave $(\omega)$ and piezoelectric coefficients of the material:

$$
[T]_{6 \times 1}=\left[C^{\prime}\right]_{6 \times 6}[S]_{6 \times 1}
$$

For the $\mathrm{ZnO}$ material, the effective elastic constant matrix is given below [49]:

$$
\left[C^{\prime}\right]=\left[\begin{array}{cccccc}
c_{11}+e_{31}^{2} Y_{33} & c_{12}+e_{31}^{2} Y_{33} & c_{13}+e_{31}^{2} Y_{33} & 0 & 0 & 0 \\
c_{12}+e_{31}^{2} Y_{33} & c_{11}+e_{31}^{2} Y_{33} & c_{23}+e_{31} e_{33} Y_{33} & 0 & 0 & 0 \\
c_{13}+e_{31} e_{33} Y_{33} & c_{23}+e_{31} e_{33} Y_{33} & c_{33}+e_{33}^{2} Y_{33} & 0 & 0 & 0 \\
0 & 0 & 0 & c_{44}+e_{15}^{2} Y_{22} & 0 & 0 \\
0 & 0 & 0 & 0 & c_{44}+e_{15}^{2} Y_{11} & 0 \\
0 & 0 & 0 & 0 & 0 & c_{66}
\end{array}\right]
$$

$e_{i j}$ are the piezoelectric coupling effect matrix elements of $\mathrm{ZnO}$ and $Y_{i j}$ are the elements of a defined matrix given below: 


$$
[Y]=\left[\begin{array}{ccc}
\frac{1}{\frac{j}{\omega} \sigma+\varepsilon_{11}} & 0 & 0 \\
0 & \frac{1}{\frac{j}{\omega} \sigma+\varepsilon_{22}} & 0 \\
0 & 0 & \frac{1}{\frac{j}{\omega} \sigma+\varepsilon_{33}}
\end{array}\right]
$$

The matrix elements of the derived effective elasticity have an imaginary part. This imaginary part will create a decaying factor in the wave vector of the travelling wave, hence taking into account the acoustoelectric relating attenuation. This attenuation is maximized at the intermediate conductivity range. Hence, we have switched the conductivity value from low to high levels, to avoid operating in the intermediate high attenuation zone, and guarantee a low loss tunable behavior for the proposed device.

\section{Results and Discussion}

We assume a PnC of $\mathrm{ZnO}$ pillars with radius of $r=65 \mathrm{~nm}$ and height of $h=100 \mathrm{~nm}$ in a square lattice form with lattice constant of $l=200 \mathrm{~nm}$, as the base platform for designing our waveguide, according to our previous work [51]. The substrate material is Si and its thickness is assumed to be five time of PnC's lattice constant [43]. Other material parameters, such as elastic and piezoelectric coupling matrices are given in our pervious study [51]. Here, we propose the elliptical CRSAW, and design the relating structural parameters so that single mode waveguiding with controlled bandwidth is allowed. Using Bloch-Floquet boundary conditions over the side walls of the perfect PnC unit cell (figure 2(a)), we have calculated the related band structure along the high symmetry points of the first Brillouin Zone (BZ), which is shown in the inset of figure 2(a). The calculated band structure is demonstrated in figure 2(b), which shows three bandgaps for SAWs inside the sound cone. The sound cone borders, separating the surface-coupled mode bands from those of bulk modes along different wave vector directions, are figured out by calculating the SAW velocity in substrate material in each direction. Two low frequency local resonance bandgaps are highlighted by green ribbons and numbered by 1 and 2, and the high frequency bandgap, highlighted by blue ribbon, is the Bragg bandgap (labeled B). Considering that the surface coupled modes and the resulting local resonance bands come from coupling the resonators (pillars) through the substrate surface in the $\mathrm{PnC}$, we can explain the emergence of some flat surface coupled bands (say, $3^{\text {th }}$ band in figure 2(b)) in comparison with other dispersive surface coupled bands (say, $4^{\text {th }}$ band in figure $2(\mathrm{~b})$ ). Generally, the surface coupled mode with smaller displacements on the substrate surface between the pillars, have weaker elastic coupling between the pillars, and show dispersive-less or flat band behavior. Hence, the coupling strength between the resonators in each surface coupled mode, at each direction plays the crucial role for the dispersion property of the band. 


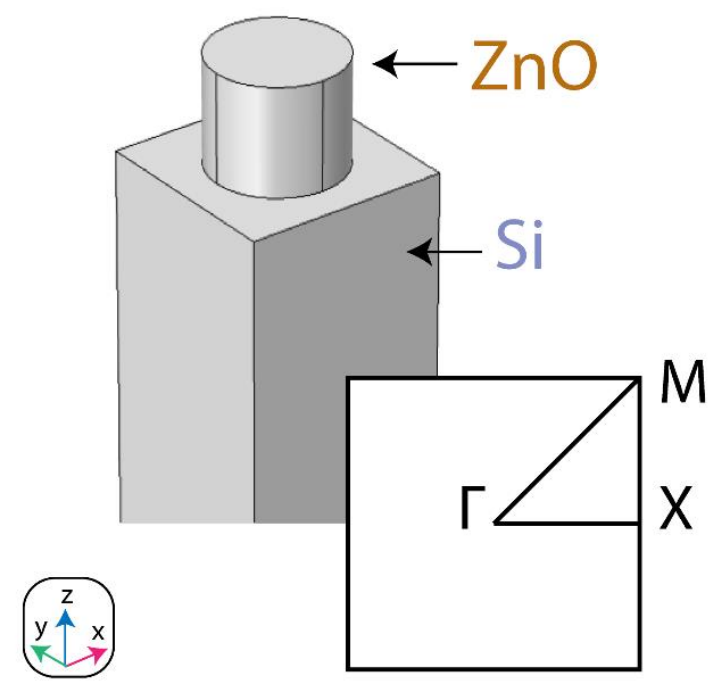

(a)

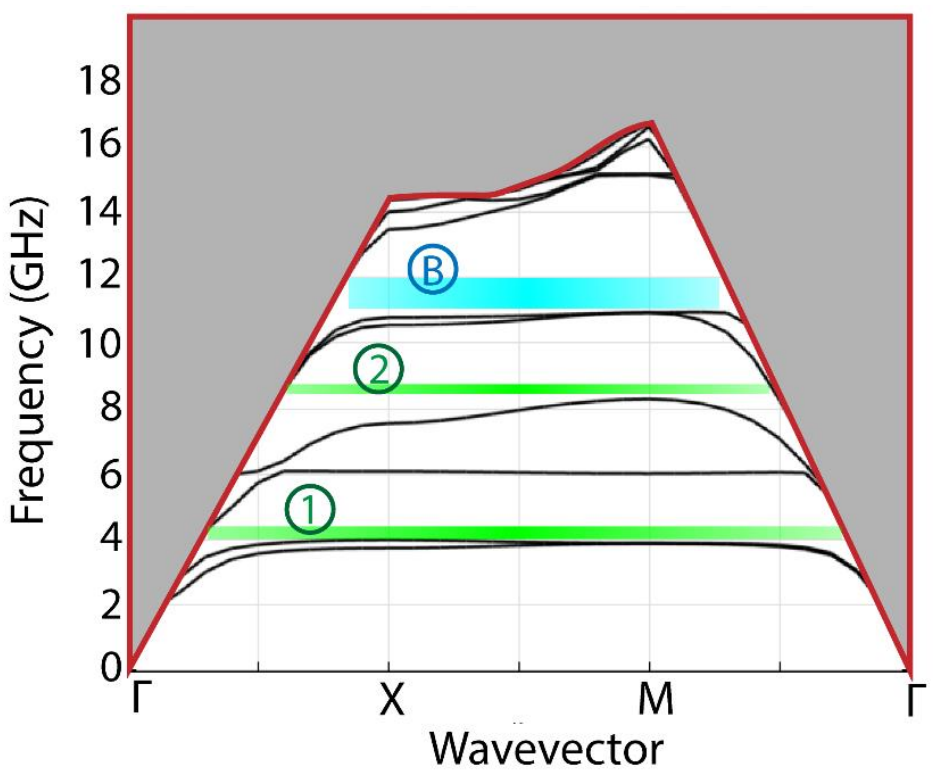

(b)

Figure 2. (a) The perfect PnC unit cell and the first BZ. (b) The perfect PnC band structure. The two green ribbons show the local resonance bandgaps $(1 \& 2)$ and the blue ribbon shows the Bragg bandgap (B).The Gray region is the sound cone which separates the surface coupled modes from the bulk modes.

In order to investigate the line defect guiding modes inside the local resonance bandgap, we have assumed a 1x11 supercell of defected $\mathrm{PnC}$, which has finite length in $\mathrm{y}$ direction and is considered to be infinite in $\mathrm{x}$ direction. By applying periodic boundary conditions in $\mathrm{x}$ direction, the band structure in $\Gamma-\mathrm{X}$ direction is calculated. Our pervious study of the pillar and hollow cylinder line defects show that thin hollow cylinder line defects can lead to a relatively high quality factor transmission inside the bandgap, since the created guiding bands are almost flat and have a very low group velocity [49]. However, inevitable creation of multiple guiding modes inside the bandgap due to the structural symmetry of the hollow cylinder, has encouraged us to break this symmetry by introducing elliptical cylinder resonators. Two radii of the elliptical cylinder are named as $a$ and $b$, which are depicted in the defected supercell geometry in figure 3(a). By choosing the bigger radius equal to the radius of the pillars i.e. $b=r=65 \mathrm{~nm}$, we have changed the radii ratio $(a / b)$ to investigate its effect on guiding modes creation. Three bandstructures for three different $a / b$ ratios of $0.3,0.45$ and 0.5 in $\Gamma$-X direction in the frequency range of the first local resonance bandgap (3-6 GHz) are shown in figure 3(b)-(d). The gray region is the sound cone and the guiding bands are shown by red bands. For small $a / b$ ratios, the elliptical cylinder creates two guiding modes inside the bandgap, while increasing $a / b$ ratio leads to frequency separation of the guiding modes. The high frequency band moves to lower frequencies and the low frequency band shifts upward in frequency. The mode shapes for the discussed guiding modes, marked as A, B, C, D, E and F near to the X point of BZ are shown in figure 3(e)-(g). The modes $\mathrm{A}$ and $\mathrm{B}$ in part (e) show a sagittal and shear polarized deformation shape for $\mathrm{b}=65 \mathrm{~nm}$ and $a / b=0.3$ at frequencies of $4.13 \mathrm{GHz}$ and $4.3 \mathrm{GHz}$, respectively. By increasing the $a / b$ ratios up to 0.45 , the modes $\mathrm{C}$ and $\mathrm{D}$ show shear and sagittal polarization deformation shapes at frequencies equal to $4.15 \mathrm{GHz}$ and 5.15 $\mathrm{GHz}$, respectively. These results show that by increasing $a / b$ from 0.3 to 0.45 , the shear mode moves to slightly lower frequencies, while the sagittal mode moves to higher frequencies. Finally, for $a / b=0.5$ the guiding frequencies corresponding to the modes $\mathrm{E}$ and $\mathrm{F}$ approach to $4.11 \mathrm{GHz}$ and $5.39 \mathrm{GHz}$ with shear and sagittal polarizations, as shown in part $(\mathrm{g})$, respectively. It is obvious that for the higher aspect ratios up to $a / b \approx 1$, both of the guiding modes merge with the PnC transmission bands completely. Figure 3 illustrates that for an elliptical cylinder with $b=65 \mathrm{~nm}$ and $a / b=0.45$, a single guiding mode exists inside the bandgap, 
close to the mid gap. Thus, we have selected this elliptical cylinder line defect to compare its guiding mode characteristics with those of previously studied pillar and hollow cylinder line defects. It is notable that in wider study of the structural parameters, we investigated $a / b$ ratios higher than 1 , which led to dominance of Rayleigh mode at the defect frequencies, and a considerable attenuation in the shear-transmission spectra. In this line of research, we also investigated the effect of elliptical cylinder rotation (for $a / b<1$ ), which resulted in a mode conversion in the defect guiding modes, so that Rayleigh components come dominant for higher rotation angles. Again, this mode conversion behavior results in more attenuation of the shear-horizontal transmission. Hence, we have utilized elliptical cylinder waveguides with $\mathrm{a} / \mathrm{b}<1$ and no rotation, to achieve the maximum shear transmission value at the defect guiding frequencies.
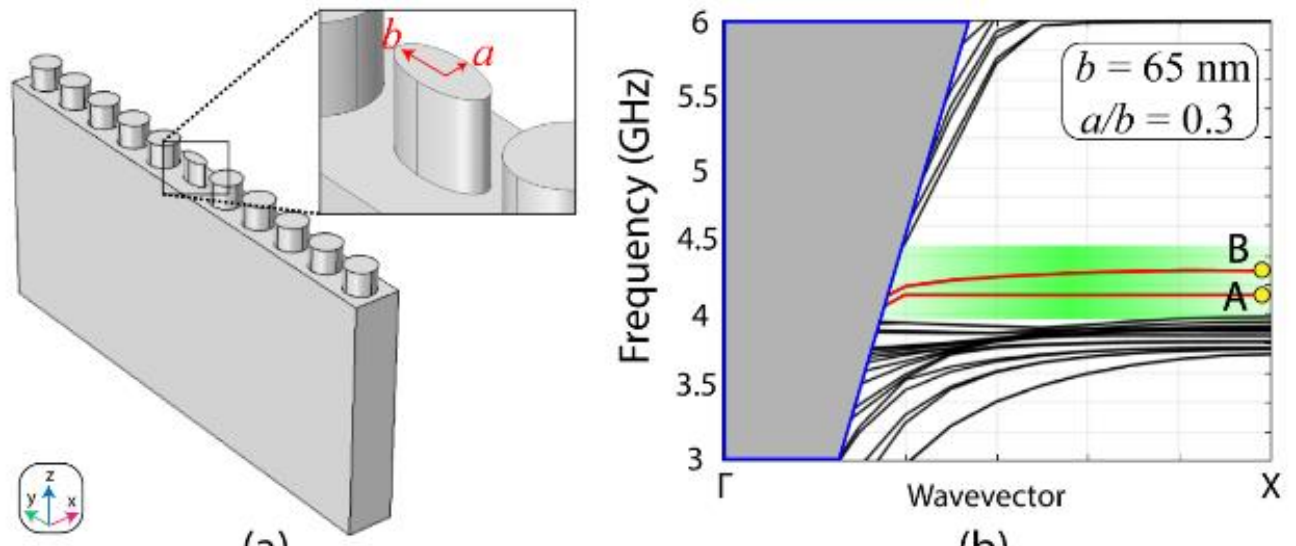

(a)

(b)

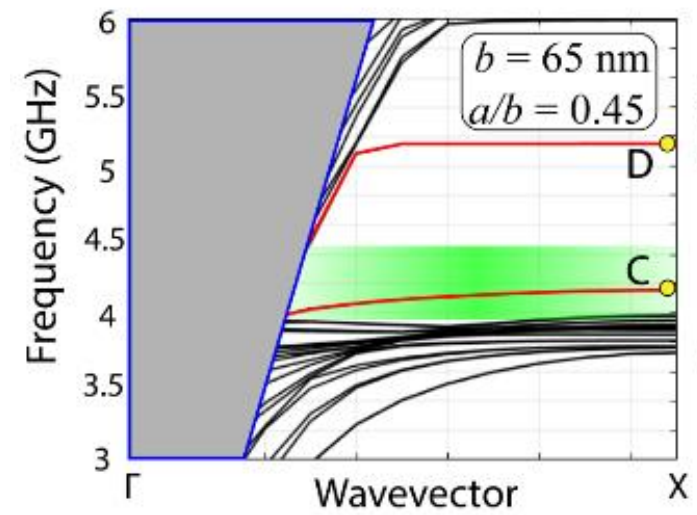

(c)

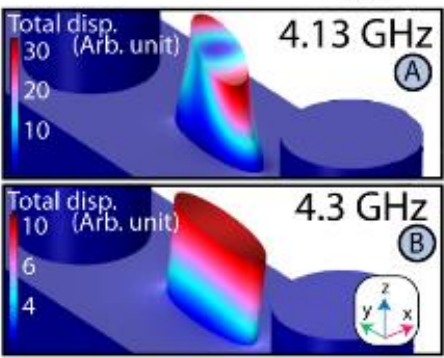

(e)

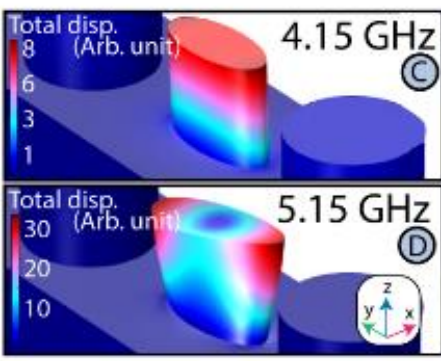

(f)

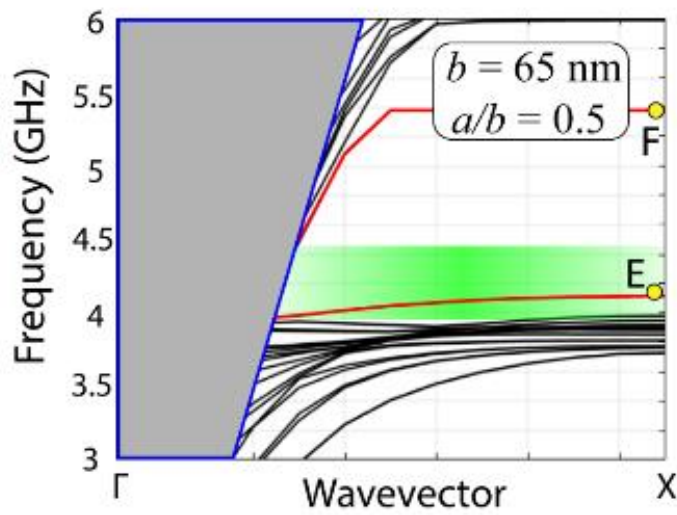

(d)

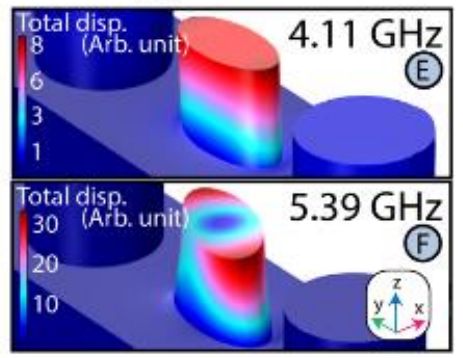

(g)

Figure 3. The defect supercell geometry with an elliptical cylinder defect. Bandstructures corresponding to the elliptical cylinder defects with $b=65 \mathrm{~nm}$, (b) $a / b=0.3$, (c) $a / b=0.45$, and (d) $a / b=0.5$. The deformation shapes of the modes, (e) A and B, (f) C and D, and (g) E and F in the bandstructures.

In order to compare the guiding mode of the selected elliptical cylinder defect (mode $\mathrm{C}$ in figure 3(c)) with pillar and hollow cylinder line defect modes, we depict the latter two related band structures in figure 4. 
Figure 4(a)-(c) show the supercell of the pillar defect with radius of $r_{d}=85 \mathrm{~nm}$, the related band structure, and the total displacement at the defect guiding frequency of $f_{d}=4.15 \mathrm{GHz}$ near the $\mathrm{X}$ point of the first $\mathrm{BZ}$. Figure 4(d)-(f), show the hollow cylinder line defect supercell with outer radius of $r_{o}=65 \mathrm{~nm}$ and inner to outer radii ratio of $r_{i} / r_{o}=0.85$, the related band structure, and the total displacement at two guiding frequency modes of $f_{d 1}=4.05 \mathrm{GHz}$ and $f_{d 2}=4.09 \mathrm{GHz}$. The details on the applied structural parameters for the pillar and hollow cylinder defects are in accordance with the optimized waveguiding behavior, given in our previous work [49]. As the results show, the guiding band of the pillar defect has a large group velocity value, which will result in a wide band transmission, almost spanning the entire bandgap width. On the other hand, the hollow cylinder defect creates two interlaced guiding bands with very low group velocity inside the bandgap. The selected elliptical cylinder waveguide has a single guiding band inside the bandgap with a group velocity value, which is higher than that of the hollow cylinder waveguide and lower than that of the pillar defect. We choose hollow cylinders and elliptical cylinders, as two candidates for low bandwidth waveguiding, and to reduce the transmission bandwidth, we introduce and study the corresponding CRSAWs.

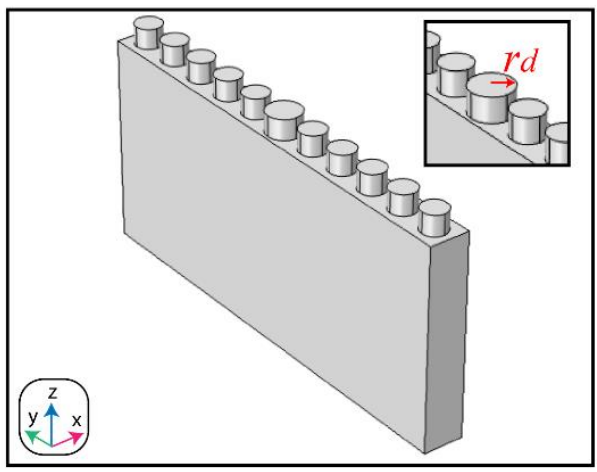

(a)

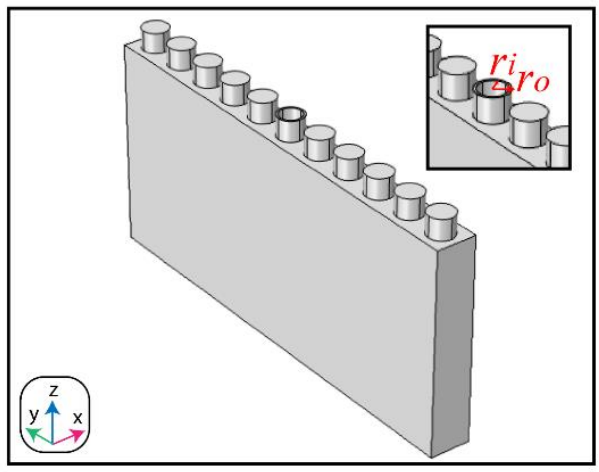

(d)

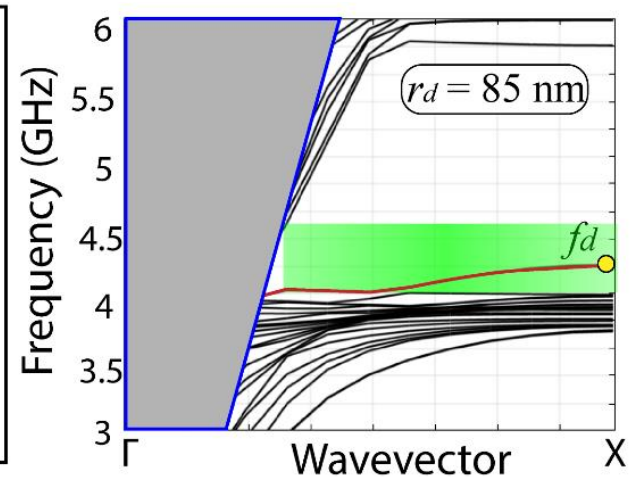

(b)

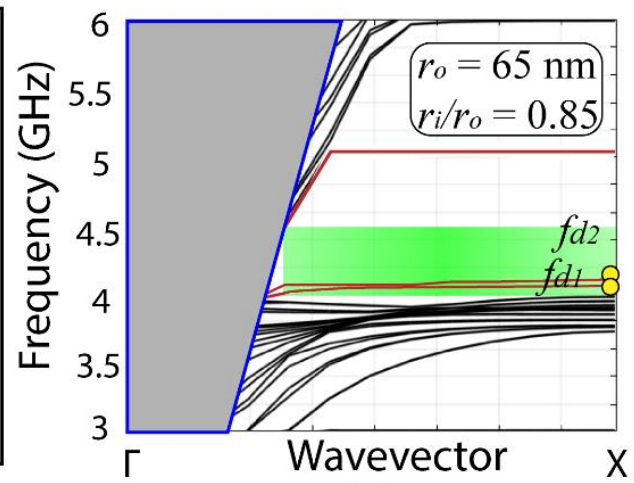

(e)

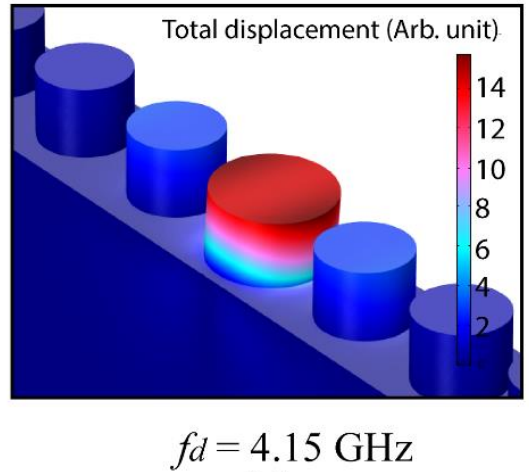

(c)

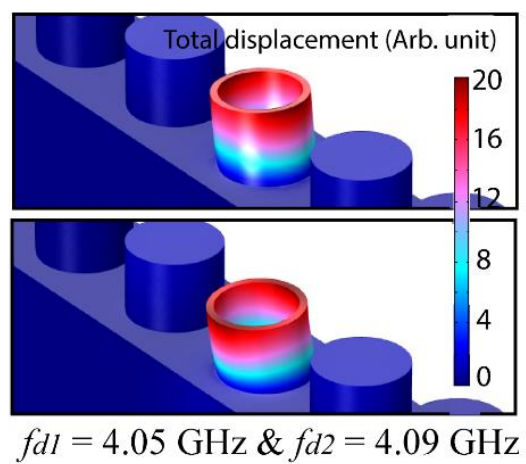

(f)

Figure 4. (a) The supercell, (b) the band structure, and (c) the total displacement for the pillar defect with $r_{d}=85 \mathrm{~nm}$, at defect frequency of $f_{d}=4.15 \mathrm{GHz}$ near to the X point. (d) The supercell, (e) the band structure, and (f) the total displacements for the hollow cylinder defect with $r_{o}=65 \mathrm{~nm}$ and $r_{i} / r_{o}=0.85$, at two guiding frequencies of $f_{d l}=4.05$ $\mathrm{GHz}$ and $f_{d 2}=4.09 \mathrm{GHz}$.

Initially, we have investigated the transmission of the elliptical and hollow cylinder waveguides for an incident shear-horizontal SAW. We have focused on the shear wave transmission, because it has been shown that the shear SAW benefits from higher velocity in comparison with the sagittal SAW, which can lead to higher energy conservation near the metallized surface of SAW transducers, and higher sensitivity for SAW based sensors [26]. It is notable that the $\mathrm{ZnO}$ structures are assumed intrinsic (low conductivity state). In order to extract the transmission spectra, we have assumed a 11x11 defected PnC, surrounded by absorbing layers and low reflection terminating boundaries. The incident SAW is triggered through a source 
cutline in front of the PnC, while the polarization of it can be determined by applying a specific amount of force in different directions (i.e. $x, y, z$ ) to this cutline. For example, shear SAW can be initiated by applying a specific amount of force only in y direction. The transmission spectra are extracted by collecting the sum of absolute values of displacement components (i.e. $\left|u_{x}\right|+\left|u_{y}\right|+\left|u_{z}\right|$ ) over the output edge and dividing it by the same value collected over the input edge, as shown in Figure 5(a).
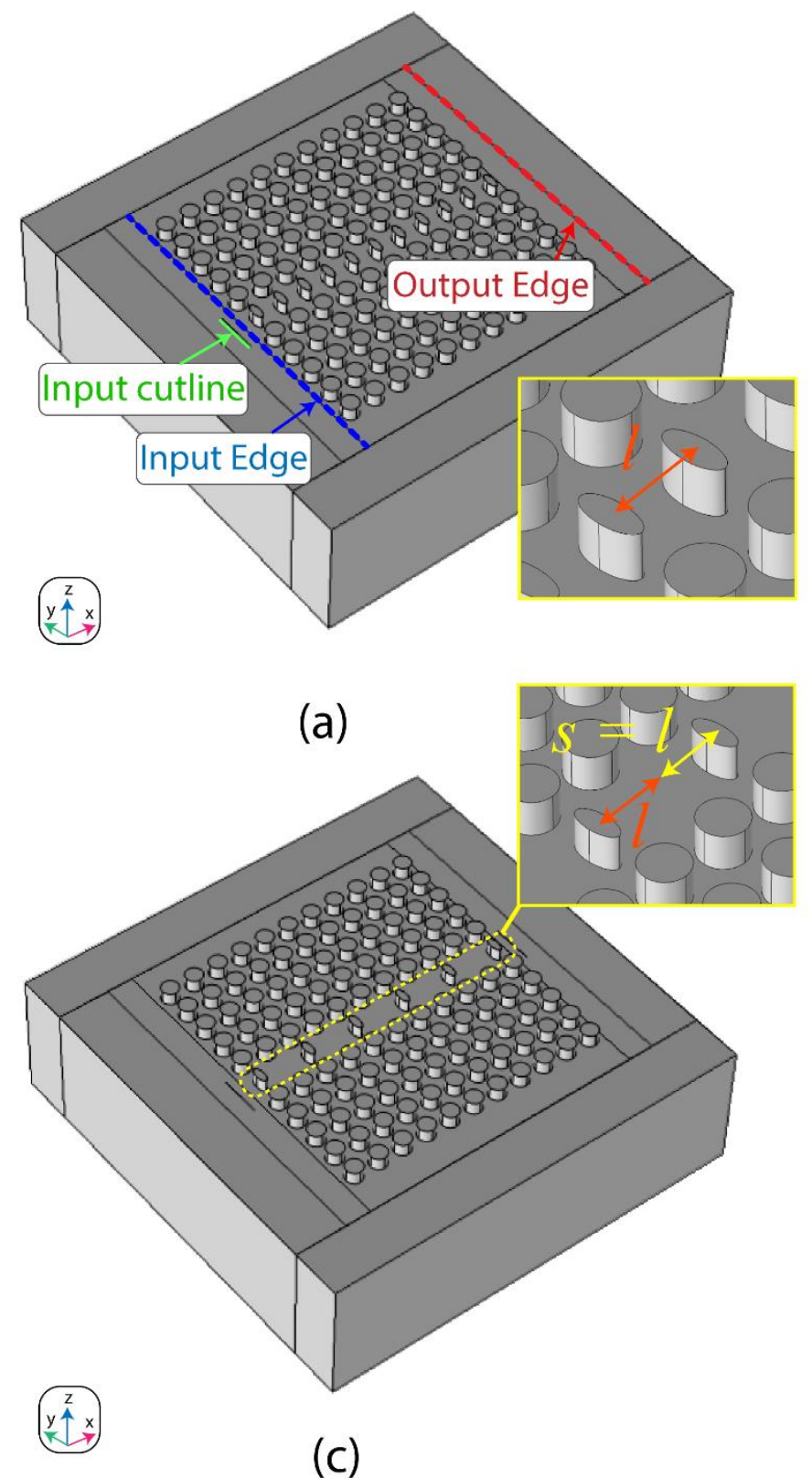

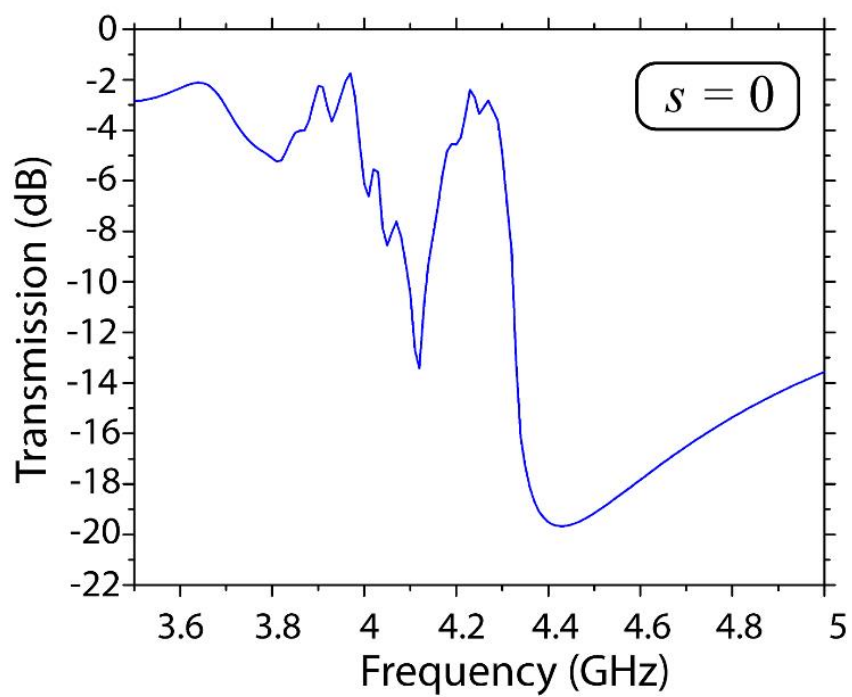

(b)

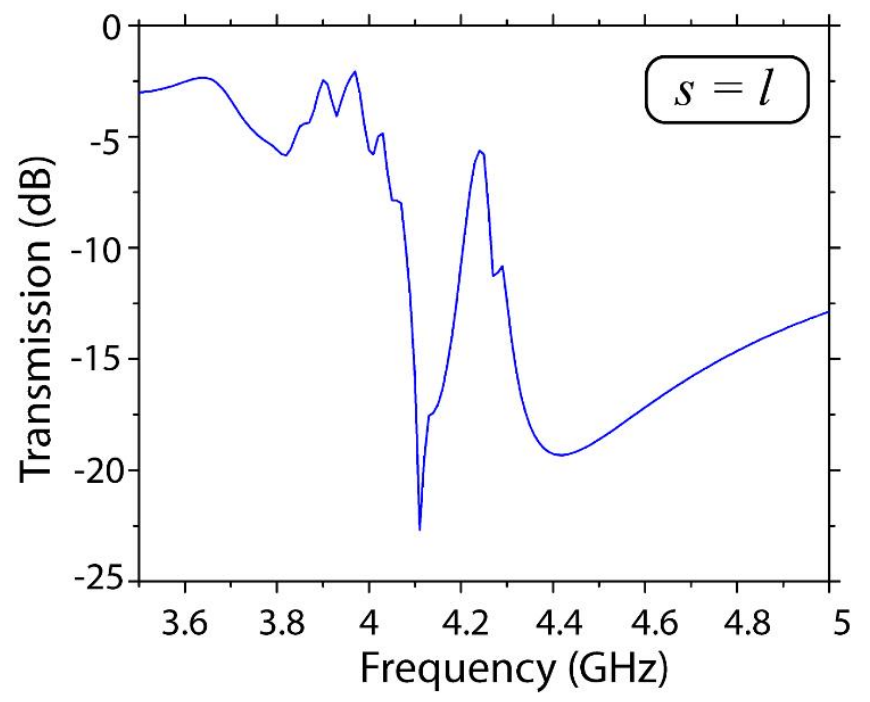

(d)

Figure 5. (a) The elliptical cylinder waveguide geometry with $b=65 \mathrm{~nm}, a / b=0.45$, and $s=0$ in a $11 \times 11 \mathrm{PnC}$ platform. Input and output cutlines are highlighted by blue and red lines. (b) Shear wave transmission related to part (a). (c) The elliptical cylinder CRSAW with $s=l$, and (d) the related shear wave transmission.

After investigating the normal elliptical and hollow cylinder waveguides, we have studied the corresponding CRSAWs to see the effect of extra spacing value $(s)$ between the line defect resonators on the FWHM and peak intensity of the transmission spectra. Figure 5(a)-(b) show the structure of an elliptical cylinder waveguide with no extra spacing $(s=0)$, and the related shear wave transmission over the first local resonance bandgap frequency range. Figure 5(c)-(d) show the structure of CRSAW with $s=l$, and the related 
shear SAW transmission. As the results show, the extra spacing has made FWHM of the line defect to drop, since the coupling among the defect resonators weakens by increasing their interspacing.

Then, to elaborate CRSAWs we have investigated the effect of varying the extra spacing for elliptical and hollow cylinder CRSAWs. All of ZnO structures for both of CRSAWs are assumed to be in their intrinsic state with no additional electrical conductivity. Considering that the tailored line defect consists of individual uniform resonators that have come near each other, we can assume the related defect band as the combination of degenerate resonance levels of these resonators. In other words, we expect that discrete resonance energy levels of the isolated resonators at larger s values, turn into a resonance guiding band with higher bandwidth at lower s values. On the other hand, since coupling strength between the defect resonators decreases for larger s values, higher loss values or lower transmission peak intensities are expected. Hence, we define a figure of merit (FOM) as the multiplication of FWHM by transmission peak intensity (dB) to find the optimum s value, so that the simultaneous lowest FWHM and highest peak intensity are achieved. Figure 6(a) shows the variations of FWHM (black, left-axis), and peak intensity (red, right-axis), while figure 6(b) illustrates FOM for the previously selected elliptical cylinder CRSAW as a function of $s /$. As figure 6(a) shows, whenever s increases from 0 to 1, FWHM of CRSAW decreases from $129 \mathrm{MHz}$ to its lowest value equal to $48 \mathrm{MHz}$ (shown in part (a) by a red circle), while the transmission peak intensity decreases from $2.42 \mathrm{~dB}$ to $-5.62 \mathrm{~dB}$. However, further increasing the extra spacing, FWHM increases slightly, while the peak intensity keeps the decreasing trend, so that the transmission peak disappears for $s=2 l$ since the resonators will not be coupled to each other at all. FOM versus $s / l$ is plotted in figure 6(b), wherein the optimum point is defined so that the lowest FWHM and the highest peak intensity are achievable simultaneously. Hence, we have chosen $s=0.5 \mathrm{l}$ as the optimum point for extra spacing of the elliptical cylinder CRSAW, in which the FWHM is equal to $69 \mathrm{MHz}$, while the peak intensity is about $-2.54 \mathrm{~dB}$. Similar study has been carried out for the hollow cylinder CRSAW and the results are shown in figure 7(a)-(b). Figure 7(a) indicates that FWHM (black, left-axis) decreases from $91 \mathrm{MHz}$ for $s=0$, to its minimum value of about $26 \mathrm{MHz}$ for $s=l$. On the other hand, the peak intensity variations (red, right-axis) of the hollow cylinder CRSAW shows an overall decreasing behavior, and drops from $-0.23 \mathrm{~dB}$ to about $-4.39 \mathrm{~dB}$ as the $s$ value increases from 0 to $l$, until it disappears for s values higher than 2l. Figure 7(b), shows a maximum value for FOM at $s=0$ for the hollow cylinder CRSAW, where FWHM =91 MHz, and the peak intensity has a value equal to $-0.23 \mathrm{~dB}$.

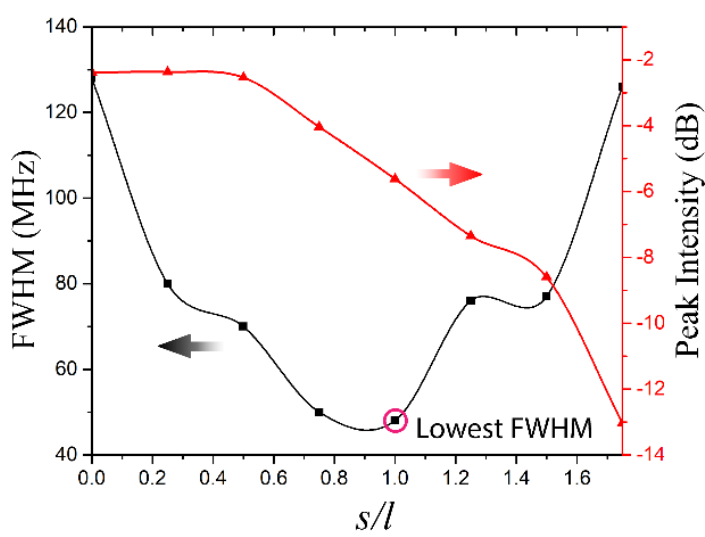

(a)

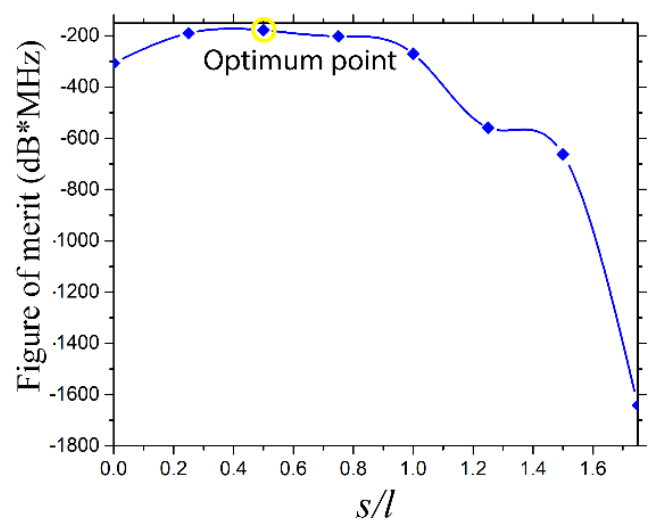

(b)

Figure 6. (a) FWHM (black, left-axis), the peak intensity (red, right-axis), and (b) FOM variations for the elliptical cylinder CRSAWs with $b=65 \mathrm{~nm}$ and $a / b=0.45$, as a function of $s / l$. 


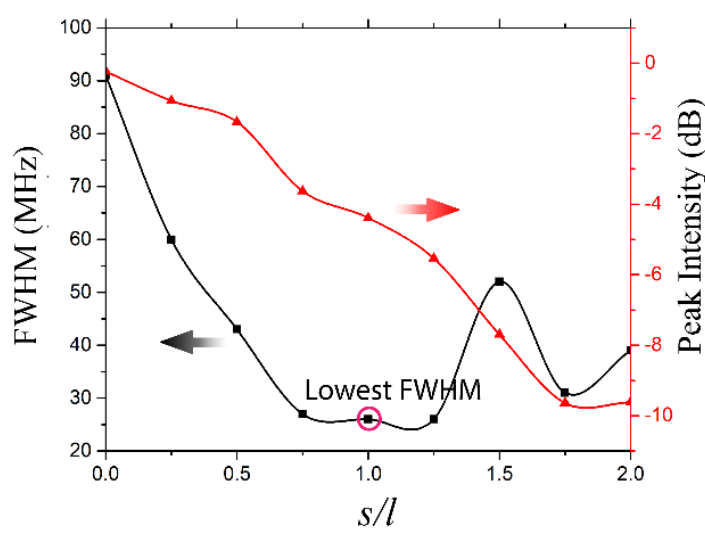

(a)

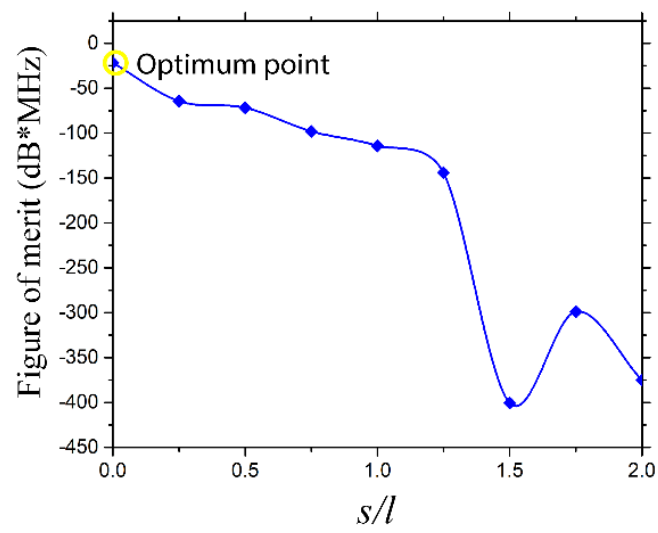

(b)

Figure 7. (a) FWHM (black, left-axis), peak intensity (red, right-axis), and (b) FOM variations of the hollow cylinder CRSAWs with $r_{o}=65 \mathrm{~nm}$ and $r_{i} / r_{o}=0.85$, as a function of $s / l$.

The hollow cylinder CRSAW shows a transmission with FWHM less than that of the elliptical cylinder CRSAW. However, the multi-mode waveguiding nature of the hollow cylinder CRSAW is not favorable for applications such as demultiplexing, since the coupling of wave between input and output waveguides would be less efficient. So, the single mode behavior of the elliptical cylinder resonators makes them suitable candidates to be used in SAW demultiplexers. Moreover, from the fabrication point of view, the elliptical cylinder resonators are easier to fabricate with lower dimension error than the thin hollow cylinder resonators, especially in nano-dimensions.

Finally, to introduce reconfigurable waveguiding behavior of the studied CRSAWs, the conductivity $(\sigma)$ of $\mathrm{ZnO}$ structures are changed from $0.01 \mathrm{~S} / \mathrm{m}$ to $100 \mathrm{~S} / \mathrm{m}$. The increment in the conductivity can relax the extra stiffness created by $\mathrm{AE}$ in $\mathrm{ZnO}$, thus change the effective elastic constant and tune the CRSAW guiding frequencies. It should be mentioned that the UV illumination depth in $\mathrm{ZnO}$ is equal to $180 \mathrm{~nm}$, which is well higher than our $\mathrm{ZnO}$ structures height. Thus we have assumed a uniform AE effect through the $\mathrm{ZnO}$ thickness, and have neglected $\mathrm{AE}$ in $\mathrm{Si}$ because of its centrosymmetric nature. The detailed $\mathrm{AE}$ equations addressing $\mathrm{ZnO}$ elasticity modulation due to UV illumination (i.e. conductivity change) are given in our pervious report [49]. It is notable that the AE loss is accounted for in this equation by the complex effective stiffness and wave number, resulting in elastic wave damping. This loss can be significant in a certain conductivity rang, which depends on the piezoelectric material properties. For $\mathrm{ZnO}$, AE loss is maximum for a conductivity range between about $1 \mathrm{~S} / \mathrm{m}$ to $10 \mathrm{~S} / \mathrm{m}$, which is well out of our utilized conductivity up/down limiting levels $(0.01 \mathrm{~S} / \mathrm{m}$ and $100 \mathrm{~S} / \mathrm{m})$ [62], to achieve the desired acoustoelectric tuning mechanism, but avoid high attenuation. Taking into account AE effect, we have calculated the bandstructures of the elliptical CRAWs with $s=0, s=0.5 l$ and $s=l$ for very low and high conductivity values, wherein AE loss become negligible, and the AE effective elastic constants approach to their frequency-independent limits. We plot the calculated bandstructures and the transmission spectra of the elliptical CRSAWs with $s=0, s=0.5 l$ and $s=l$ in figure 8. Figure 8(a), (b) and (c) exhibit the overlaid low and high conductivity $(\sigma)$ bandstructures for the elliptical cylinder CRSAWs with $s=0, s=0.5 l$ and $s=l$, respectively. The guiding bands are shown with black dashed and solid red lines for low and high $\sigma$ values and the bandgap region is shown by green ribbon. Also, figure 8(d), (e) and (f) show the corresponding shear SAW transmission spectra of the elliptical cylinder CRSAWs with $s=0, s=0.5 l$ and $s=l$ for $\sigma=0.01 \mathrm{~S} / \mathrm{m}$ (black dashed curve) and $\sigma=100 \mathrm{~S} / \mathrm{m}$ (red curve), respectively $(b=65 \mathrm{~nm}, a / b=0.45)$. By increasing $\sigma$ from $0.01 \mathrm{~S} / \mathrm{m}$ to $100 \mathrm{~S} / \mathrm{m}$, for $s=0$, the guiding frequency shifts from $f_{d 0}=4.23 \mathrm{GHz}$ to $f^{\prime}{ }^{\prime} 0=4.14 \mathrm{GHz}\left(\Delta f_{\mathrm{d}}=-90 \mathrm{MHz}\right)$, while FWHM decreases from $129 \mathrm{MHz}$ to $116 \mathrm{MHz}$ (equal to $10 \%$ improvement). For $s=0.5 l$ case, the guiding frequency shifts from $f_{d 1}=4.26 \mathrm{GHz}$ to $f^{\prime} d 1=$ 
4.14 GHz $\left(\Delta f_{\mathrm{d}}=-120 \mathrm{MHz}\right)$ and FWHM decreases from $69 \mathrm{MHz}$ to $55 \mathrm{MHz}$ (equal to $21 \%$ improvement). Finally, for $s=l$, the guiding frequency shifts from $f_{d 2}=4.24 \mathrm{GHz}$ to $f^{\prime}{ }_{d 2}=4.12 \mathrm{GHz}\left(\Delta f_{\mathrm{d}}=-120 \mathrm{MHz}\right)$ and FWHM decreases from $48 \mathrm{MHz}$ to $40 \mathrm{MHz}$ (equal to $16 \%$ improvement). It should be noted that our calculated transmission spectra confirm significant attenuation for the intermediate conductivity values (for instance $\sigma \approx 2 \mathrm{~S} / \mathrm{m}$ ) of $\mathrm{ZnO}$, due to the significant $\mathrm{AE}$ loss in this regime.

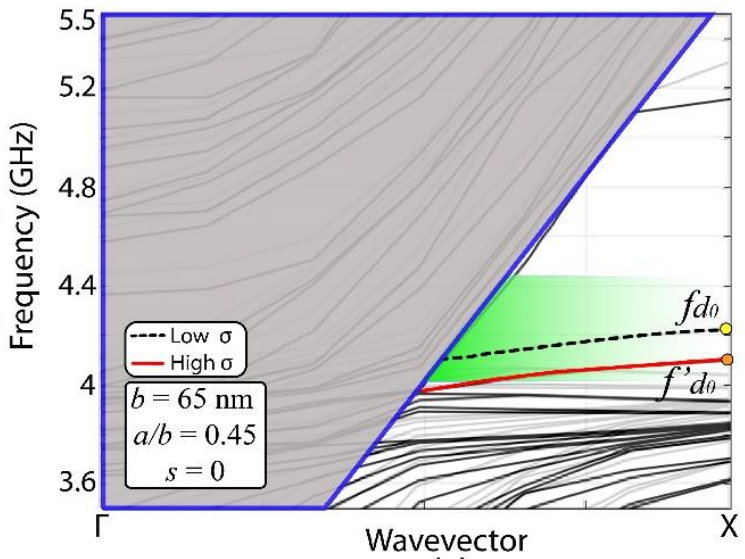

(a)

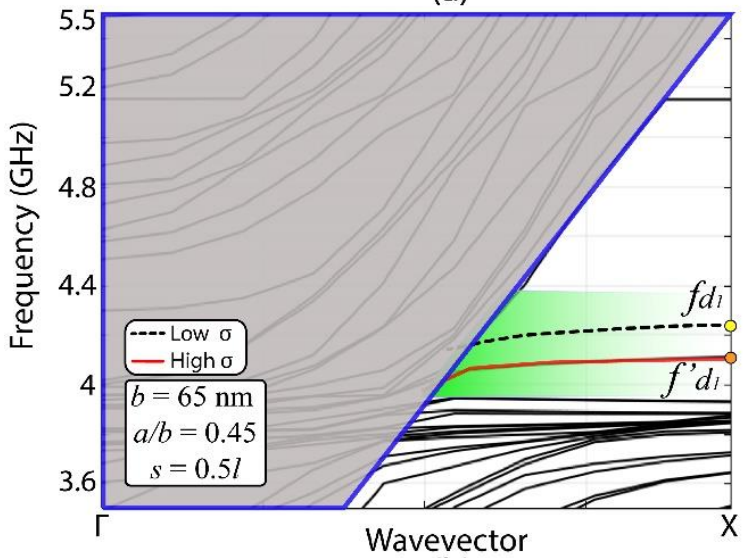

(b)

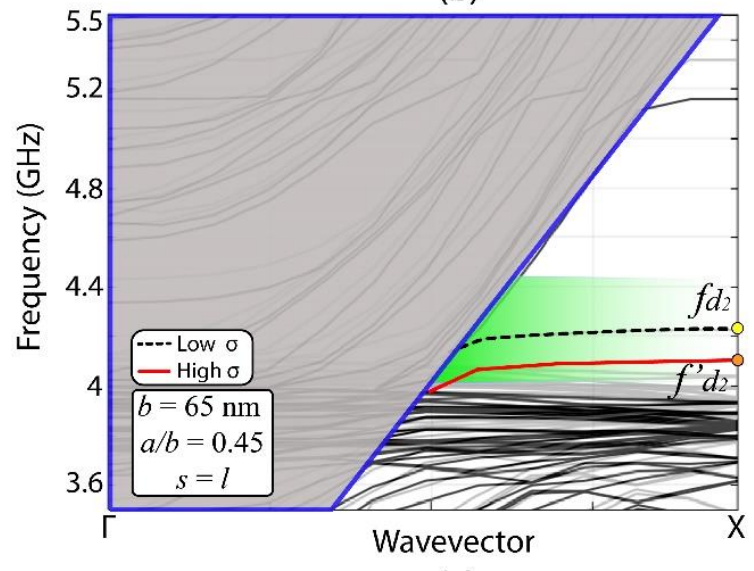

(c)
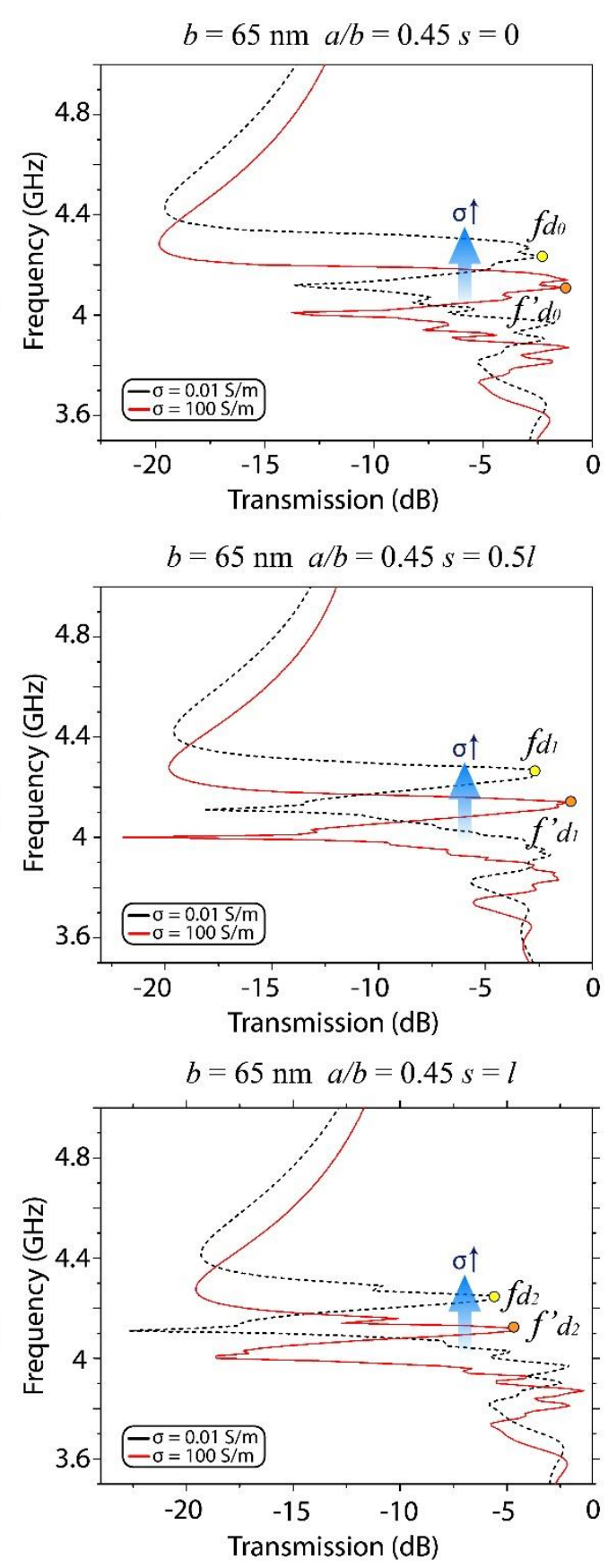

Figure 8. The overlaid low and high conductivity $(\sigma)$ bandstructures for the elliptical cylinder CRSAWs with $b=65$ $\mathrm{nm}$ and $a / b=0.45$ for: (a) $s=0$, (b) $s=0.5 l$ and (c) $s=l$. Shear SAW transmission spectra for elliptical cylinder CRSAWs with: (d) $s=0$, (e) $s=0.5 l$ and (f) $s=l$ for $\sigma=0.01 \mathrm{~S} / \mathrm{m}$ (black dashed curve) and $\sigma=100 \mathrm{~S} / \mathrm{m}$ (red solid curve), while $b=65 \mathrm{~nm}$ and $a / b=0.45$. 
The summary of the frequency and FWHM modulations of the designed elliptical cylinder CRSAWs with $b$ $=65 \mathrm{~nm}$ and $a / b=0.45$, for three different $s$ values, as a consequence of conductivity switching $(\sigma=0.01 \mathrm{~S} / \mathrm{m}$ and $100 \mathrm{~S} / \mathrm{m}$ ), are given in Table 1 . These results show that a maximum frequency shift of $120 \mathrm{MHz}$ and a FWHM change percentage of $21 \%$ is achieved for $s=0.5 \mathrm{l}$ by increasing the conductivity to $100 \mathrm{~S} / \mathrm{m}$. Thus, a significant enhancement of about $57 \%$ is achieved for FWHM of the optimized elliptical cylinder CRSAW, owing to both increasing $s$ value from 0 to $0.5 l$, and increasing $\sigma$ from $0.01 \mathrm{~S} / \mathrm{m}$ to $100 \mathrm{~S} / \mathrm{m}$.

\begin{tabular}{|c|c|c|c|c|}
\hline$s$ & $f_{d}$ & $\% \Delta f_{d}$ & FWHM & $\% \Delta$ FWHM \\
\hline 0 & $4.23 \mathrm{GHz}$ & $-2.12 \%$ & $129 \mathrm{MHz}$ & $-10 \%$ \\
\hline $0.5 l$ & $4.26 \mathrm{GHz}$ & $-2.81 \%$ & $69 \mathrm{MHz}$ & $-21 \%$ \\
\hline$l$ & $4.24 \mathrm{GHz}$ & $-2.83 \%$ & $48 \mathrm{MHz}$ & $-16 \%$ \\
\hline
\end{tabular}

Table 1. Low conductivity guiding frequencies $\left(f_{d}\right)$, relative frequency shifts $\left(\% \Delta f_{d}\right)$, low conductivity FWHM and relative FWHM variation (\% $\%$ FWHM) of the elliptical cylinder with $b=65 \mathrm{~nm}$ and $a / b=0.45$, for $s=0,0.5 l$, and $l$, when changing conductivity from $0.01 \mathrm{~S} / \mathrm{m}$ to $100 \mathrm{~S} / \mathrm{m}$.

Herein, we proposed a CRSAW structure based on elliptical cylinders, as a reconfigurable low bandwidth SAW filter or waveguide, in which FWHM and guiding frequency can be tuned by switching the conductivity of the $\mathrm{ZnO}$ structures. The proposed SAW waveguide benefits from a simple fabrication process, beside low bandwidth and single mode behavior, which make it an attractive building block for RF telecommunication applications, such as SAW filters, demultiplexers, and duplexers.

\section{Conclusion}

We propose a bandwidth tunable elliptical cylinder CRSAW inside a $\mathrm{PnC}$ of $\mathrm{ZnO}$ pillars over a $\mathrm{Si}$, based on a local resonance bandgap, which creates a single guiding band with reduced transmission bandwidth. The observed dispersive behavior of this guiding mode and the consequent transmission bandwidth is optimized by controlling the mechanical coupling between defect resonators. Precisely, we demonstrate that the bandwidth characteristic can be engineered by changing the spacing between the defect resonators. Our optimized CRSAW design with $s=0.5 \mathrm{l}$ led to $47 \%$ decrement in the transmission bandwidth (from $129 \mathrm{MHz}$ to $69 \mathrm{MHz}$ ), without involving significant transmission loss or fabrication complication. Furthermore, by changing the conductivity of $\mathrm{ZnO}$ structures from $0.01 \mathrm{~S} / \mathrm{m}$ to $100 \mathrm{~S} / \mathrm{m}$, we have modulated the effective elasticity, which induces a frequency shift of $\Delta f_{d}=-120 \mathrm{MHz}$, and $\Delta \mathrm{FWHM}=-21 \%$ for the designed elliptical CRSAW with $s=0.5 \mathrm{l}$. Finally, we achieve 57\% improvement for FWHM value of the proposed CRSAW by increasing conductivity of $\mathrm{ZnO}$ structures to $100 \mathrm{~S} / \mathrm{m}$. The proposed engineered low bandwidth and single mode reconfigurable CRSAW seems a promising building block for miniature and efficient SAW RF-filters, demultiplexers, and Mach-Zehnder devices for wireless communication applications.

\section{Acknlowdgment}

The authors acknowledge the partial financial support of the Iran National Science Foundation, and the financial support from Tarbiat Modares University through grant \#IG-39703.

\section{References}

[1] Lewis M 1982 SAW filters employing interdigitated interdigital transducers IIDT Ultrason. Sym. 12.

[2] Ruppel C W 2017 Acoustic wave filter technology - A Review IEEE Trans. Ultrason. Ferroele. Freq. Cont. 642690905.

[3] Shao L et al 2019 Phononic Band Structure Engineering for High-Q Gigahertz Surface Acoustic Wave Resonators on Lithium Niobate Phys. Rev. Applied 12014022. 
[4] Hikita M, Ishida Y, Tabuchi T and Kurosawa K 1988 Miniature SAW antenna duplexer for 800-MHz portable telephone used in cellular radio systems IEEE Trans. Microwave Theory Tech. 361047.

[5] Tsutsumi J et al 2003 in IEEE Frequency Control Symp. 861.

[6] Kadota M et al 2005 Surface Acoustic Wave Duplexer for US Personal Communication Services with Good Temperature Characteristics Jpn. J. Appl. Phys. 44 6B.

[7] Rana L, Gupta R, Tomar M and Gupt V 2017 ZnO/ST-Quartz SAW resonator: An efficient NO2 gas sensor Sens. Actu. B: Chem. 252840.

[8] Sadek A Z et al 2007 A ZnO nanorod based layered ZnO/64' YX LiNbO3 SAW hydrogen gas sensor Thin Solid films 515 8705-8708.

[9] Nakamura K 2007 Shear-Horizontal piezoelectric surface acoustic waves Jpn. J. Appl. Phys. 46442.

[10] Narayanamurti V, Störmer H L, Chin M A, Gossard A C and Wiegmann W 1979 Selective Transmission of High-Frequency Phonons by a Superlattice: The "Dielectric" Phonon Filter Phys. Rev. Lett. 432012.

[11] Sigalas M and Economou E N 1993 Band structure of elastic waves in two dimensional systems Solid State Commun. 86141. [12] Kushwaha M S, Halevi P, Dobrzynski L and Djafari-Rouhani B 1993 Acoustic band structure of periodic elastic composites Phys. Rev. Lett. 712022.

[13] Olsson R H et al 2011 Micro and Nano Fabricated Phononic Crystals: Technology and Applications Proc. IEEE Int. Ultrason. Symp. (IUS) 983.

[14] Mohammadi S, Eftekhar A A, Khelif A, Hunt W and Adibi A 2008 Evidence of large high frequency complete phononic band gaps in silicon phononic crystal plates Appl. Phys. Lett. 92221905.

[15] Delpero T, Schoenwald S, Zemp A and Bergamini A 2016 Structural engineering of three-dimensional phononic crystals Journal of Sound and Vibration 363 156-165.

[16] Yu D, Wen J, Zhao H, Liu Y and Wen X 2018 Vibration reduction by using the idea of phononic crystals in a pipe-conveying fluid Journal of Sound and Vibration 318 193-205.

[17] Wang Y, Wang T, Liang J, Wang Y and Laude V 2018 Channeled spectrum in the transmission of phononic crystal waveguides Journal of Sound and Vibration 437 410-421.

[18] M. Ghasemi Baboly et al 2016 Demonstration of acoustic waveguiding and tight bending in phononic crystals Appl. Phys. Lett. 109183504.

[19] Pourabolghasem R, Dehghannasiri R, Eftekhar A A and Adibi A 2018 Waveguiding Effect in the Gigahertz Frequency Range in Pillar-based Phononic-Crystal Slabs Phys. Rev. Applied 9014013.

[20] Huang Zi-Gui 2011 Silicon-based filters, resonators and acoustic channels with phononic crystal structures J. Phys. D Appl. Phys. 4424.

[21] Shakeri A, Darbari S and Moravvej-Farshi MK 2019 Designing A tunable acoustic resonator based on defect modes stimulated by selectively biased PZT rods in a 2D phononic crystal Ultrasonics 928.

[22] Javadi M, Heidari A and Darbari S 2018 Realization of enhanced sound-driven CNT-based triboelectric nanogenerator, utilizing sonic array configuration $\mathrm{Cu}$. Appl. Phys. 18361.

[23] H. Shin et al 2015 Control of coherent information via on-chip photonic-phononic emitter-receivers Nat. Commun. 6 6427.

[24] Pennec Y, Djafari-Rouhani B, Vasseur J O, Khelif A and Deymier P A 2004 Tunable filtering and demultiplexing in phononic crystals with hollow cylinders Phys. Rev. E. 69046608.

[25] Rostami-Dogolsara B, Moravvej-Farshi M and Nazari F 2016 Designing switchable phononic crystal-based acoustic demultiplexer IEEE Trans. Ultrason. Ferroele. Freq. Cont. 631468.

[26] Lucklum R 2014 Phononic crystals and metamaterials - promising new sensor platforms Procedia. Eng. 8740.

[27] Zhang X, Jackson T, Lafond E, Deymier P and Vasseur J 2006 Evidence of surface acoustic wave band gaps in the phononic crystals created on thin plates Appl. Phys. Lett. 88041911.

[28] Kokkonen K, Kaivola M, Benchabane S, Khelif A and Laude V 2007 Scattering of surface acoustic waves by a phononic crystal revealed by heterodyne interferometry Appl. Phys. Lett. vol. 91083517.

[29] Tian Y, Li H, Ke Y, Yuan C and He S 2016 P-Matrix, Analysis of Surface Acoustic Waves in Piezoelectric Phononic Crystals IEEE Trans. Ultrason. Ferroele. Freq. Cont. 63757. 
[30] Dehghannasiri R, Eftekhar A A and Adibi A 2018 Hypersonic Surface Phononic Bandgap Demonstration in a CMOSCompatible Pillar-Based Piezoelectric Structure on Silicon Phys. Rev. Applied 10064019.

[31] S. Benchabane et al 2017 Surface-Wave Coupling to Single Phononic Subwavelength Resonators Phys. Rev. Applied 8 034016.

[32] L. Shao et al 2019 Phononic Band Structure Engineering for High-Q Gigahertz Surface Acoustic Wave Resonators on Lithium Niobate Phys. Rev. Applied 12014022.

[33] Z. Liu et al 2000 Locally resonant sonic materials Science 2895485.

[34] C. Goffaux et al 2002 Evidence of Fano-Like Interference phenomena in locally resonant materials Phys. Rev. Lett. 88225502.

[35] Pennec Y, Djafari-Rouhani B, Larabi H, Vasseur J O and Hladky-Hennion A C 2008 Low-frequency gaps in a phononic crystal constituted of cylindrical dots deposited on a thin homogeneous plate Phys. Rev. B 78104105.

[36] Khelif A, Achaoui Y, Benchabane S, Laude V and Aoubiza B 2010 Locally resonant surface acoustic wave band gaps in a two-dimensional phononic crystal of pillars on a surface Phys. Rev. B 81214303.

[37] Achaoui Y, Khelif A, Benchabane S, Robert L and Laude V 2011 Experimental observation of locally-resonant and Bragg band gaps for surface guided waves in a phononic crystal of pillars Phys. Rev. B 83104201.

[38] Ash B, Worsfold S, Vukusic P and Nash G 2017 A Highly attenuating and frequency tailorable annular hole phononic crystal for surface acoustic waves, Nat. Commun. 8174.

[39] Ash B Ph.D. Thesis University of Exeter, 2018.

[40] Muzar E, Azodi Aval G and Stotz James A H 2018 Wet-etched phononic crystal waveguiding on GaAs J. Phys. D Appl. Phys. 514.

[41] Cicek A, Gungor T, Adem Kaya O and Ulug B 2015 Guiding airborne sound through surface modes of a two-dimensional phononic crystal J. Phys. D Appl. Phys. 4823.

[42] Zhang D et al 2017 Investigation of surface acoustic wave propagation in composite pillar based phononic crystals within both local resonance and Bragg scattering mechanism regimes J. Phys. D Appl. Phys.50 43.

[43] Guo Y, Schubert M and Dekorsy T 2016 Finite element analysis of surface modes in phononic crystal waveguides J. Appl. Phys. 119124302.

[44] Hutson and White D 1962 Elastic Wave propagation in piezoelectric semiconductors J. Appl. Phys. 3340.

[45] Parmenter R 1953 The Acousto-Electric Effect Phys. Rev. 89990.

[46] Emanetoglu N W et al 2004 Surface acoustic wave ultraviolet photodetectors using epitaxial ZnO multilayers grown on $\mathrm{r}$ plane sapphire Appl. Phys. Lett. 853702.

[47] Chivukula V, Ciplys D, Shur M and Dutta P 2010 ZnO nanoparticle surface acoustic wave UV sensor Appl. Phys. Lett. 96 233512.

[48] Guo Y J et al 2015 Ultraviolet sensing based on nanostructured ZnO/Si surface acoustic wave devices Sm. Mat. Struc. 24 125015.

[49] Ishii Y, Sasaki R, Nii Y, Ito T, and Onose Y 2018 Magnetically Controlled surface acoustic waves on multiferroic BiFeO3 Phys. Rev. Applied 9034034.

[50] Verba R et al 2018 Nonreciprocal surface acoustic waves in multilayers with magnetoelastic and interfacial DzyaloshinskiiMoriya interactions Phys. Rev. Applied 9064014.

[51] Taleb F and Darbari S 2019 Tunable Locally Resonant Surface-Acoustic-Waveguiding Behavior by Acoustoelectric Interaction in ZnO-Based Phononic Crystal Phys. Rev. Applied 11024030.

[52] Taleb F, Darbari S and Khelif A 2021 Reconfigurable locally resonant surface acoustic demultiplexing behavior in ZnO-based phononic crystal J. Appl. Phys 129024901.

[53] A. Khelif et al 2003 Trapping and guiding of acoustic waves by defect modes in a full-band-gap ultrasonic crystal Phys. Rev. B 68214301 .

[54] Khelif A, Mohammadi S, Eftekhar A A, Adibi A and Aoubiza B 2010 Acoustic confinement and waveguiding with a linedefect structure in phononic crystal slabs J. Appl. Phys. 1084515.

[55] Sainidou R, Stefanou N and Modinos A 2006 Linear chain of weakly coupled defects in a three-dimensional phononic crystal: A model acoustic waveguide Phys. Rev. B 74172302. 
[56] Zhang M, Zhong W and Zhang X 2012 Defect-free localized modes and coupled-resonator acoustic waveguides constructed in two-dimensional phononic quasicrystals J. Appl. Phys. 111104314.

[57] Escalante J M, Martınez A and Laude V 2013 Dispersion relation of coupled-resonator acoustic waveguides formed by defect cavities in a phononic crystal J. Phys. D: Appl. Phys. 4647.

[58] Yariv A, Xu Y, Lee R K and Scherer A 1999 Coupled-resonator optical waveguide: a proposal and analysis Opt. Lett. 24711.

[59] Xu Y, Lee R K, Yariv A 2000 Propagation and second-harmonic generation of electromagnetic waves in a coupled-resonator optical waveguide J. Opt. Soc. Am. B 17387.

[60] S. Olivier et al 2001 Miniband transmission in a photonic crystal coupled-resonator optical waveguide Opt. Lett. 261019.

[61] Xia F, Sekaric L, O’Boyle M and Vlasov Y 2006 Coupled resonator optical waveguides based on silicon-on-insulator photonic wires Appl. Phys. Lett. 89041122.

[62] Fu C, Lee K J, Lee K and Yang S S 2015 Low-intensity ultraviolet detection using a surface acoustic-wave sensor with a Agdoped ZnO nanoparticle film Smart Mater. Struct. 24015010. 